\title{
ORIGINAL ARTICLE \\ Pharmacodynamics and effectiveness of topical nitroglycerin at lowering blood pressure during autonomic dysreflexia
}

\author{
R Solinsky ${ }^{1,2,3}$, AE Bunnell ${ }^{3,4}$, TA Linsenmeyer ${ }^{1,2,5}$, JN Svircev ${ }^{3,6}$, A Engle ${ }^{7}$ and SP Burns $s^{3,6}$
}

Study design: Secondary analysis of prospectively collected observational data assessing the safety of an autonomic dysreflexia (AD) management protocol.

Objectives: To estimate the time to onset of action, time to full clinical effect (sustained systolic blood pressure (SBP) $<160 \mathrm{~mm} \mathrm{Hg}$ ) and effectiveness of nitroglycerin ointment at lowering blood pressure for patients with spinal cord injuries experiencing AD.

Setting: US Veterans Affairs inpatient spinal cord injury (SCI) unit.

Methods: Episodes of AD recalcitrant to nonpharmacologic interventions that were given one to two inches of $2 \%$ topical nitroglycerin ointment were recorded. Pharmacodynamics as above and predictive characteristics (through a mixed multivariate logistic regression model) were calculated.

Results: A total of 260 episodes of pharmacologically managed AD were recorded in 56 individuals. Time to onset of action for nitroglycerin ointment was 9-11 min. Time to full clinical effect was 14-20 min. Topical nitroglycerin controlled SBP $<160 \mathrm{~mm} \mathrm{Hg}$ in $77.3 \%$ of pharmacologically treated $A D$ episodes with the remainder requiring additional antihypertensive medications. $A$ multivariate logistic regression model was unable to identify statistically significant factors to predict which patients would respond to nitroglycerin ointment (odds ratios 95\% confidence intervals 0.29-4.93). The adverse event rate, entirely attributed to hypotension, was 3.6\% with seven of the eight events resolving with close observation alone and one episode requiring normal saline.

Conclusions: Nitroglycerin ointment has a rapid onset of action and time to full clinical effect with high efficacy and relatively low adverse event rate for patients with SCI experiencing AD.

Spinal Cord (2017) 55, 911-914; doi:10.1038/sc.2017.58; published online 6 June 2017

\section{INTRODUCTION}

Autonomic dysreflexia (AD) is a potentially life-threatening condition, which occurs in patients with spinal cord injury (SCI). In this condition, an unmitigated stimulus below the level of injury leads to deregulated sympathetic outflow and a potentially dangerous, progressive rise in blood pressure. Even though $\mathrm{AD}$ is potentially dangerous, it commonly occurs in the inpatient and outpatient setting for those at risk (classically SCI at or above T6). ${ }^{1}$ When first-line nonpharmacologic measures are ineffective at quelling the rise in blood pressure, pharmacologic options are typically employed. ${ }^{2}$ Despite its recommendation in the Consortium for Spinal Cord Medicine's clinical practice guideline as a potential management agent for $\mathrm{AD}$ with systolic blood pressure (SBP) over $150 \mathrm{~mm} \mathrm{Hg}$, ${ }^{3}$ we are not aware of any studies that have explored the pharmacodynamics of topical nitroglycerin for $\mathrm{AD}$ in SCI patients. Topical nitroglycerin ointment leads to vasodilation through smooth muscle relaxation and, in neurologically intact individuals, has been shown to have an onset of action of 20-30 min with peak effect taking an estimated 60 min. ${ }^{4,5}$ This study was designed to evaluate the use of topical nitroglycerin as management for $\mathrm{AD}$ in patients with SCI.

\section{MATERIALS AND METHODS}

This is a secondary analysis from the data set of a previously published safety and effectiveness study. ${ }^{6}$ In that institutional review board-approved study, a structured $\mathrm{AD}$ management protocol encompassing progressive escalation of nonpharmacologic management, topical nitroglycerin and then further antihypertensive administration was employed on an inpatient Veterans Affairs SCI unit as outlined in the Consortium for Spinal Cord Medicine's clinical practice guideline. Nonpharmacologic management included sitting a patient up, loosening constrictive garments, and assessing for noxious stimuli from the bladder, bowels and skin before further escalation to pharmacologic management starting with topical nitroglycerin. Topical $2 \%$ nitroglycerin ointment was chosen as the first-line antihypertensive pharmacologic agent as it can be easily removed when blood pressure decreases to within a safe target range. During each episode of $\mathrm{AD}$, nursing staff prospectively recorded data on a paper $\log$ sheet designed for the safety and efficacy study.

For this study, an episode of $\mathrm{AD}$ was defined as (1) signs or symptoms of $\mathrm{AD}$ with SBP >150 $\mathrm{mm} \mathrm{Hg}$ or (2) SBP elevated above $150 \mathrm{~mm} \mathrm{Hg}$, as measured on routine vital sign checks without symptoms (given the high rate of silent dysreflexia). ${ }^{7}$ Following failure of nonpharmacologic management at lowering blood pressure, 1 inch $(25.4 \mathrm{~mm})$ of topical nitroglycerin ointment was applied to a standardized measuring template and placed on the upper chest, regardless of level of injury. For this study, nitroglycerin ointment was considered to show

\footnotetext{
${ }^{1}$ Kessler Institute for Rehabilitation, West Orange, NJ, USA; ${ }^{2}$ Department of Physical Medicine and Rehabilitation, Rutgers New Jersey Medical School, Newark, NJ, USA; ${ }^{3}$ Department of Rehabilitation Medicine, University of Washington, Seattle, WA, USA; ${ }^{4}$ Harborview Medical Center, Seattle, WA, USA; ${ }^{5}$ Department of Surgery, Division of Urology, Rutgers New Jersey Medical School, Newark, NJ, USA; ${ }^{6}$ Spinal Cord Injury Service, Veterans Affairs Puget Sound Health Care System, Seattle, WA, USA and ${ }^{7}$ Department of Statistics, University of Washington, Seattle, WA, USA

Correspondence: Dr R Solinsky, Department of Physical Medicine and Rehabilitation, Rutgers New Jersey Medical School, 90 Bergen Street, Suite 3200, Newark, NJ 07103-1709, USA.

E-mail: ryanjs2@uw.edu

Received 1 February 2017; revised 17 March 2017; accepted 18 April 2017; published online 6 June 2017
} 
effectiveness if it lowered SBP below the target range of $<160 \mathrm{~mm} \mathrm{Hg}$. If SBP was not reduced to $<160 \mathrm{~mm} \mathrm{Hg}$ within $10 \mathrm{~min}$, a second inch of nitroglycerin ointment was applied to the measuring template and placed on the upper chest. Patients were monitored closely, and if after an additional $10 \mathrm{~min}, \mathrm{SBP}$ was not maintained below the target range, further escalation with alternative antihypertensives was employed (see previous study for full protocol details). ${ }^{6} \mathrm{AD}$ episodes were considered controlled when SBP persisted $<160 \mathrm{~mm} \mathrm{Hg}$ during subsequent vitals over the next $3 \mathrm{~h}$. Adverse events were defined as SBP $<70 \mathrm{~mm} \mathrm{Hg}$, need for intravenous medication or related medical instability necessitating transfer off the SCI unit. Monitoring for serious adverse events was also undertaken.

During the $3 \frac{1}{2}$ year period, all episodes of $\mathrm{AD}$ occurring in the inpatient SCI unit were prospectively recorded by nursing staff. Episodes of $\mathrm{AD}$ were included in this substudy if (1) nonpharmacologic measures had failed to effectively lower SBP $<160 \mathrm{~mm} \mathrm{Hg}$, (2) no additional antihypertensive medications were given at the same time as nitroglycerin ointment outside of the protocol, and (3) time points were recorded for topical nitroglycerin application, $10 \mathrm{~mm} \mathrm{Hg}$ decrease in SBP, and time to reach sustained decrease in SBP below the target threshold.

\section{Statistical analysis}

Descriptive statistics were calculated for both episodes of $\mathrm{AD}$ and individual patient demographics using Excel (Version 2013, Microsoft Corporation, Redmond, WA, USA). Mean initial presenting vitals (SBP, diastolic blood pressure, heart rate) and adverse events were calculated for each step in the protocol and as an aggregate. An unpaired t-test was used to compare presenting vitals between episodes that resolved at each step of management, with $P$-values $<0.05$ regarded as statistically significant. Time to onset of action was defined as the time it took for SBP to decrease $10 \mathrm{~mm} \mathrm{Hg}$ after topical nitroglycerin application. Time to full clinical effect was defined as the time it took for SBP to decrease $<160 \mathrm{~mm} \mathrm{Hg}$ after topical nitroglycerin application.

A mixed multivariate logistic regression model was generated using the statistical software R (version 3.1.3, package.lme4, The R Foundation, Vienna, Austria) to identify potential factors influencing if topical nitroglycerin was effective at reaching the target blood pressure (SBP $<160 \mathrm{~mm} \mathrm{Hg}$ ). In this way, we aimed to determine predictors to guide if practitioners should proceed directly on to alternative antihypertensives. For this model, episodes that were controlled with one or two inches of topical nitroglycerin ointment were grouped as responsive to the medication. Independent variables for the regression model included the American Spinal Injury Association Impairment Scale grades as administered at the time of data capture, ${ }^{8}$ neurological level of injury (as grouped in Table 1), initial vital signs (SBP, diastolic blood pressure, heart rate) and cause of injury (traumatic vs non-traumatic). Dependent variables included age and number of years since SCI. The statistical regression model utilized mixed modeling to account for potential correlation within patients who had multiple $\mathrm{AD}$ events recorded (allowing for clustered variability).

\section{Table 1 Patient demographics}

\begin{tabular}{|c|c|c|c|}
\hline \multicolumn{2}{|c|}{$\begin{array}{c}\text { Patient demographics } \\
\text { Gender }\end{array}$} & \multicolumn{2}{|l|}{ Initial cause of injury } \\
\hline \multirow[t]{3}{*}{ Male } & \multirow[t]{2}{*}{$56(100 \%)$} & Transport & $23(41.1 \%)$ \\
\hline & & Sports & $11(19.6 \%)$ \\
\hline & AIS & Fall & $9(16.1 \%)$ \\
\hline A & $36(64.3 \%)$ & Non-traumatic spinal cord dysfunction & $5(8.9 \%)$ \\
\hline$B$ & $11(19.6 \%)$ & Other traumatic causes & $4(7.1 \%)$ \\
\hline C & $5(8.9 \%)$ & Assault & $4(7.1 \%)$ \\
\hline $\mathrm{D}$ & $4(7.1 \%)$ & & \\
\hline \multicolumn{2}{|c|}{ Neurological level of injury } & \multicolumn{2}{|c|}{ Years since injury, $N=55$} \\
\hline $\mathrm{C} 1-\mathrm{C} 4$ & $13(23.2 \%)$ & $0-5$ years & $16(29.1 \%)$ \\
\hline $\mathrm{C} 5-\mathrm{C} 8$ & $33(58.9 \%)$ & $6-10$ years & $3(5.5 \%)$ \\
\hline \multirow[t]{2}{*}{$\mathrm{T} 1-\mathrm{T} 6$} & $10(17.9 \%)$ & $>10$ years & $36(65.5 \%)$ \\
\hline & & Mean & 19.6 years \\
\hline \multicolumn{2}{|c|}{ Mean age \pm s.d. (min, max) } & \multicolumn{2}{|l|}{$54 \pm 14$ years $(25,80)$} \\
\hline
\end{tabular}

Abbreviation: AIS, American Spinal Injury Association Impairment Scale grade.

$N=56$ unless otherwise noted.

\section{RESULTS}

During our study period, 445 episodes of $\mathrm{AD}$ were recorded by nursing on the inpatient SCI unit. Of these episodes, 78 resolved with nonpharmacologic management alone, 86 utilized alternative medications (benzodiazepines, opioids, etc.) along with nitroglycerin ointment, and 21 contained incomplete data on timing of vital signs or medication administration. Previous analysis of this data set had demonstrated the low concurrence of essential hypertension falsely appearing as recorded $\mathrm{AD}$ episodes. ${ }^{6}$ The remaining 260 episodes of $\mathrm{AD}$ were managed with one or two inches of nitroglycerin ointment alone before further possible escalation of the pharmacologic management protocol to administer additional antihypertensives. Summaries of descriptive statistics for each progressive step in the $\mathrm{AD}$ management appear in Table 2.

Presenting vitals were not significantly different for episodes that resolved with each step in management ( $P$-values from 0.50 to 0.55 ). If the $\mathrm{AD}$ episode responded to one or two inches of nitroglycerin ointment, the mean time to onset of action ( $10 \mathrm{~mm} \mathrm{Hg}$ drop in SBP) was $10 \mathrm{~min}$. Further details on time to onset of action are found in Figure 1. The mean time to achieve target blood pressure $(<160 \mathrm{~mm} \mathrm{Hg})$ with one to two inches of nitroglycerin ointment was $16 \mathrm{~min}$. The first inch of nitroglycerin ointment was effective at reaching the target SBP in $43.8 \%$ of episodes. The cumulative efficacy increased to $77.3 \%$ when a second inch of nitroglycerin ointment was applied.

The multivariate logistic regression demonstrated a relatively strong fit to the available data, with a graphing of Pearson residuals versus fitted values closely aligning to zero. Typically, a well-powered statistical model requires 10 episodes for each predictor. Our sample of 260 episodes greatly exceeds this requirement for our eight model predictors. When controlling for each variable, no statistically significant factors were identified to predict response to topical nitroglycerin from presenting characteristics. Full details of the associations between presenting characteristics of the patient/AD episode in relation to blood pressure control (sustained SBP $<160 \mathrm{~mm} \mathrm{Hg}$ ) with one or two inches of nitroglycerin ointment appear in Table 3.

Eight adverse events were recorded (all due to SBP $<70 \mathrm{~mm} \mathrm{Hg}$ ). Seven events resolved with close observation alone, one required a one liter of normal saline bolus (after one inch of nitroglycerin ointment). There were no serious adverse events.

\section{DISCUSSION}

This retrospective review of 260 episodes of $\mathrm{AD}$ is the first study to focus specifically on the role and pharmacodynamics of topical nitroglycerin in management of $\mathrm{AD}$. We found that topical nitroglycerin demonstrates initial effects of lowering SBP 9-11 min after being applied for AD. SBP was maintained $<160 \mathrm{~mm} \mathrm{Hg}$ 14-20 min after application and led to clinical blood pressure control in $77.3 \%$ of events, which required pharmacologic intervention. Adverse events occurred at a low rate, resolving almost entirely with observation alone, and no serious adverse events occurred.

While it is known that topical nitroglycerin works well for hypertensive crises in individuals without $\mathrm{SCI},{ }^{4}$ this study builds the literature basis for strong, isolated efficacy of topical nitroglycerin in management of $\mathrm{AD}$. Previous studies of the pharmacodynamics of topical nitroglycerin have been based on neonatal limb ischemia ${ }^{5}$ or as prophylaxis for hypertensive events prior to intubation ${ }^{9}$ or electroconvulsive therapies. ${ }^{10}$ The prophylactic nature of the latter of these studies makes it difficult to isolate when the medication begins to 
Table 2 Descriptive statistics for each step in autonomic dysreflexia management

\begin{tabular}{|c|c|c|c|c|c|c|c|c|}
\hline & \multirow{2}{*}{$\begin{array}{l}\text { Episodes resolving } \\
\text { with this step }\end{array}$} & \multirow{2}{*}{$\begin{array}{c}\text { Mean initial SBP/DBP } \\
(\mathrm{mm} \mathrm{Hg})\end{array}$} & \multirow{2}{*}{$\begin{array}{l}\text { Mean initial HR } \\
\text { (b.p.m.) }\end{array}$} & \multicolumn{2}{|c|}{ Time after nitroglycerin to: ( $\mathrm{min})$} & \multirow{2}{*}{$\begin{array}{c}\text { Mean SBP } \downarrow \\
(m m ~ H g)\end{array}$} & \multirow{2}{*}{ 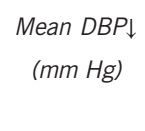 } & \multirow{2}{*}{$\begin{array}{l}A E \\
\text { rate }\end{array}$} \\
\hline & & & & $10 \mathrm{~mm} \mathrm{Hg} \mathrm{SBP \downarrow}$ & $\downarrow<160 \mathrm{~mm} \mathrm{Hg}$ & & & \\
\hline Second 1 inch nitroglycerin & 79 & 193.9/106.3 & 78.6 & 11 & 20 & 87.9 & 49.9 & $3.8 \%$ \\
\hline $\begin{array}{l}\text { Additional antihypertensive } \\
\text { medications }\end{array}$ & 67 & $188.0 / 104.8$ & 77.9 & 20 & 33 & 69.3 & 45.1 & $1.5 \%$ \\
\hline
\end{tabular}

Abbreviations: AE, adverse event; b.p.m., beats per min; DBP, diastolic blood pressure; HR, heart rate; SBP, systolic blood pressure.

'Additional antihypertensive medications' represents those episodes that received 2 inches of nitroglycerin ointment without blood pressure control and required additional antihypertensives. $N=260$.

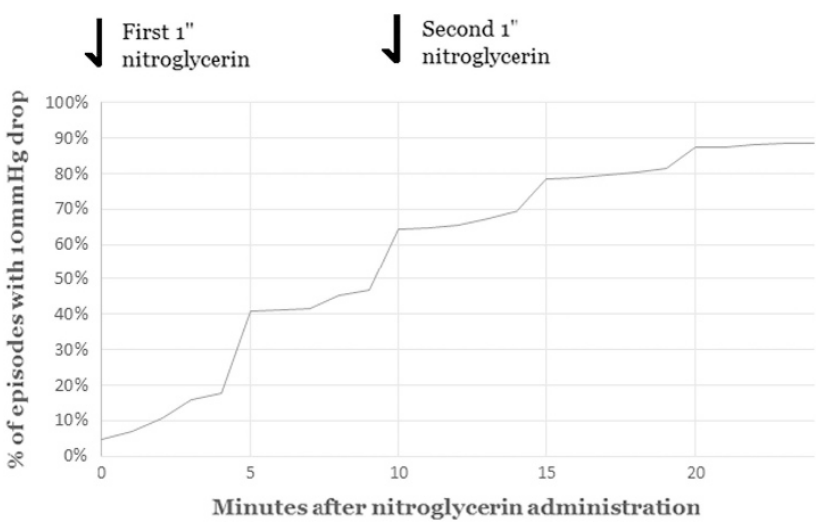

Figure 1 Percentage of AD episodes with a $10 \mathrm{~mm} \mathrm{Hg}$ decrease in SBP (time to onset of action) versus time after nitroglycerin ointment administration. A full color version of this figure is available at the Spinal Cord journal online.

clinically effect blood pressure, especially in cases of altered physiology such as SCI.

The onset of action of approximately $10 \mathrm{~min}$ (as defined by a $10 \mathrm{~mm} \mathrm{Hg}$ decrease in SBP) is likely a cautious estimate in the SCI population. The effects from prior nonpharmacologic interventions such as catheter change/digital stimulation and the original noxious stimuli may further contribute sympathetic tone during this time, leading to variable ongoing vasoconstrictive drive that the medication must counteract. While the uncontrolled nature of retrospective review does not allow isolating the effects of nitroglycerin ointment in this population, this may be a closer representation to the real-world environment and what practitioners might expect when progressing from nonpharmacologic to pharmacologic management for $\mathrm{AD}$.

Our multivariate logistic regression model compared those who had blood pressure control of $\mathrm{AD}$ following topical nitroglycerin versus those who required escalation to additional antihypertensives. This model did not reveal any significant associations with regard to American Spinal Injury Association Impairment Scale grade, neurological level of injury, initial vital signs, SCI etiology, age or duration of injury. The lack of significant predictive factors coupled with the relatively low adverse event rate (3.6\% overall), demonstrates evidence that nitroglycerin ointment appears to be effective and safe for individuals with $\mathrm{AD}$. Further, when one inch of nitroglycerin ointment fails to bring blood pressure to within safe ranges, there is still a significant benefit to adding a second inch. Given the potential for
Table 3 Odds ratios per initial autonomic dysreflexia characteristics

\begin{tabular}{|c|c|c|c|}
\hline & & Odds ratio & $95 \% \mathrm{Cl}$ \\
\hline \multicolumn{4}{|l|}{ 3A) } \\
\hline \multirow[t]{4}{*}{ AIS } & A & Reference & \\
\hline & B & 0.80 & $0.34-1.87$ \\
\hline & C & 0.99 & $0.42-2.32$ \\
\hline & D & 2.10 & $0.90-4.93$ \\
\hline \multirow[t]{3}{*}{$\mathrm{NLI}$} & $\mathrm{C} 1-\mathrm{C} 4$ & Reference & \\
\hline & $\mathrm{C} 5-\mathrm{C} 8$ & 0.68 & $0.29-1.59$ \\
\hline & T1-T6 & 1.93 & $0.83-4.53$ \\
\hline \multirow[t]{2}{*}{ Traumatic } & Yes & Reference & \\
\hline & No & 1.13 & $0.48-2.64$ \\
\hline \multicolumn{4}{|l|}{ 3B) } \\
\hline Initial SBP (1 mm Hg) & Mean $=189.5$ & 0.75 & $0.32-1.77$ \\
\hline Initial DBP (1 mm Hg) & Mean $=104.3$ & 1.41 & $0.60-3.31$ \\
\hline Initial HR (1 b.p.m.) & Mean $=76.6$ & 1.41 & $0.60-3.30$ \\
\hline Age (years) & Mean $=54.1$ & 1.57 & $0.67-3.69$ \\
\hline Injury duration (years) & Mean $=19.6$ & 0.95 & $0.41-2.24$ \\
\hline
\end{tabular}

Abbreviations: AIS, American Spinal Injury Associations (ASIA) Impairment Scale; b.p.m., beats per min; $\mathrm{Cl}$, confidence interval; DBP, diastolic blood pressure; HR, heart rate; $\mathrm{NLI}$, neurological level of injury; SBP, systolic blood pressure.

(A) Odds ratios with Wald-type $95 \% \mathrm{Cls}$ for each initial variable in predicting blood pressure control during autonomic dysreflexia with one or two inches of nitroglycerin ointment. (B) Each scaled variable represents the odds ratio per a given unit (in parenthesis) in deviation from the mean.

hypotension, possibly precipitated by leaving the topical nitroglycerin on past when clinically required, it is advised to monitor patients closely during management and remove ointment when clinically appropriate.

\section{Study limitations}

The limitations of this study stem from its observational nature and the consequent inability to control for variables. Further, our entire study population was male and tended to have more chronic injuries. Given the regimented timing of medications within our AD management protocol, it is unclear how many episodes of $\mathrm{AD}$ would have been controlled following one inch of nitroglycerin ointment, if $>10$ min was given between medications. Similarly, those episodes that were controlled within the first several minutes following the first inch of nitroglycerin ointment administration may be so due to the combined result of nonpharmacologic management. The lack of timing data on nonpharmacologic interventions limits assessing how much of a confounding factor this may have been. The stepwise response seen in Figure 1, with improved relative responsiveness at 5, 
10, 15 and $20 \mathrm{~min}$, likely represents simplification in reporting of timing data from nursing staff (rounding to the nearest $5 \mathrm{~min}$ increment) and not an underlying pharmacodynamic process. These stratified data points may suggest the degree of accuracy in how frequently recordings of timing data occurred.

Future prospective studies are warranted to elucidate the precise pharmacodynamics of topical nitroglycerin for $\mathrm{AD}$. However, this work presents strong evidence supporting the use of topical nitroglycerin ointment in real-world clinical scenarios to achieve rapid decreases in blood pressure in patients with $\mathrm{AD}$ within a clinically relevant time frame.

\section{CONCLUSION}

For required pharmacologic management of $\mathrm{AD}$ in patients with $\mathrm{SCI}$, use of first-line nitroglycerin ointment demonstrates an onset of action of 9-11 min with high attainment of target SBP and a low rate of adverse events. Given the potential risk for hypotension, close monitoring of blood pressure is warranted following application.

\section{DATA ARCHIVING}

There were no data to deposit.

\section{CONFLICT OF INTEREST}

The authors declare no conflict of interest.

\section{ACKNOWLEDGEMENTS}

This work was supported with resources and use of facilities at the VA Puget Sound Health Care System. The contents do not represent the views of the US Department of Veterans Affairs or the US Government.

1 Hubli M, Krassioukov AV. Ambulatory blood pressure monitoring in spinal cord injury: clinical practicability. J Neurotrauma 2014; 31: 789-797.

2 Karlsson AK. Autonomic dysreflexia. Spinal Cord 1999; 37: 383-391.

3 Linsenmeyer TA, Baker ER, Cardenas DD, Mobley T, Perkash I, Vogel LC. Acute management of autonomic dysreflexia: individuals with spinal cord injury presenting to health-care facilities. Clinical Practice Guidelines Consortium for Spinal Cord Medicine. Paralyzed Veterans of America, Washington DC, USA, 2001, pp 1-25.

4 Elkayam U, Aronow WS. Glyceryl trinitrate (nitroglycerin) ointment and isosorbide dinitrate: a review of their pharmacological properties and therapeutic use. Drugs 1982; 23: $165-194$

5 Wong AF, McCulloch LM, Sola A. Treatment of peripheral tissue ischemia with topical nitroglycerin ointment in neonates. J Pediatr 1992; 121: 980-983.

6 Solinsky R, Svircev JN, James JJ, Burns SP, Bunnell AE. A retrospective review of safety using a nursing driven protocol for autonomic dysreflexia in patients with spinal cord injuries. J Spinal Cord Med 2016; 39: 713-719.

7 Linsenmeyer TA, Campagnolo DI, Chou IH. Silent autonomic dysreflexia during voiding in men with spinal cord injuries. J Urol 1996; 155: 519-522.

8 Maynard FM Jr, Bracken MB, Creasey G, Ditunno JF Jr, Donovan WH, Ducker TB et al. International standards for neurological and functional classification of spinal cord injury. Spinal Cord 1997; 35: 266-274.

9 Kamra S, Wig J, Sapru RP. Topical nitroglycerin: a safeguard against pressor responses to tracheal intubation. Anaesthesia 1986; 41: 1087-1091.

10 Parab AL, Chaudhari LS, Apte J. Use of nitroglycerin ointment to prevent hypertensive response during electroconvulsive therapy-a study of 50 cases. J Postgrad Med 1992; 38: 55-57. 